https://doi.org/10.18778/2196-8403.2020.05

FELIX LEMPP

\title{
„Teppiche sind aus Geschichten gewoben“. Pro- blematisierungen generationalen Erzählens in NINO HARATISCHWILIS Das achte Leben (Für Brilka) und JETTE STECKELS Inszenierung am Thalia Theater Hamburg
}

In NinO HARATISCHWILIS Roman Das achte Leben (Für Brilka) versucht die Erzählerin Niza vergeblich, die sowjetische Geschichte Georgiens und die individuelle Geschichte ihrer Familie zwischen 1900 und 2006 aufeinander bezogen darzustellen. Der Aufsatz argumentiert, dass in den Defiziten des Erzählprogramms Nizas Widersprüche einer für den Generationenroman typischen Erzählpoetik produktiv gemacht werden, der es nicht gelingt, individuelle, familiäre und politische Geschichte zu einem sinnstiftenden Narrativ zu verbinden. Am Ende des Romans steht deshalb ein Medienwechsel von der literarischen Geschichtsdarstellung zu ihrer performativen Verkörperung im Tanz, in dem die theatrale Adaption des Romans, wie sie Jette SteCKel 2017 am Thalia Theater Hamburg inszenierte, bereits angelegt ist.

Schlüsselwörter: NinO Haratischwili, JeTte STECKel, Generationenroman, theatrale Adaption, deutschssprachige Gegenwartsliteratur, deutschsprachiges Gegenwartstheater

"Carpets are woven from stories". Problems of generational storytelling in NiNO HARATISCHWiLI's Das achte Leben (Für Brilka) and JeTte STECKEL'S adaptation at the Thalia Theater Hamburg

In NinO HaRATischwili's Das achte Leben (Für Brilka) the novel's narrator, Niza, unsuccessfully attempts to relate Georgia's Soviet history and her family's history between 1900 and 2006. The paper argues that the deficits in Niza's narrative programme are actually productive contradictions, which are typical of the narrative poetics of the generational novel, in that it does not succeed in synthesising the individual, familial and political histories into one meaningful narrative. The end of the novel therefore changes to a new medium: from the literary representation of history, to its performative 
embodiment in dance. This anticipates the theatrical adaptation of the novel by JETTE STECKEL at the Thalia Theater Hamburg in 2017.

Keywords: Nino Haratischwili, JetTe Steckel, Family Saga, Theatrical Adaptation, Contemporary German Literature, Contemporary German Theatre

\section{„Dywany utkane są z historii”. Problematyzaja narracji pokoleniowych w powieści NinO HARATISCHWILI Das achte Leben (Für Brilka) oraz inscenizacji JETTY STECKEL w Teatrze Thalia w Hamburgu}

W powieści Nino HARATISCHWILI Das achte Leben (Für Brilka) (Ósme życie - dla Brilki) narratorka Niza podejmuje nieudane próby przedstawienia radzieckiej historii Gruzji oraz związanej z nią indywidualnej historii swojej własnej rodziny w latach 1900-2006. Artykuł dostrzega w deficytach opowiadania Nizy sprzeczności. Wynikają one z wykorzystania poetyki narracyjnej, charakterystycznej dla powieści pokoleniowej, której nie udaje się powiązać ze sobą historii indywidualnej, rodzinnej i politycznej w jedno, mające wspólny sens opowiadanie. Koniec powieści posługuje się w związku z tym nowym medium: rezygnuje $\mathrm{z}$ literackiego opowiadania o historii i przechodzi do jej performatywnego ucieleśnienia w tańcu. Antycypuje ono adaptację teatralną, jaką była w 2017 r. inscenizacja JeTTy STECKEL w Teatrze Thalia w Hamburgu.

Słowa klucze: NinO HARATISCHWILI, JETTE STECKEL, powieść pokoleniowa, adaptacja teatralna, niemiecka literatura współczesna, niemiecki teatr współczesny

Die Zeitspanne, die Nino NINO HARATISCHWILIS dritter Roman Das achte Leben (Für Brilka) abdeckt, ist einschüchternd: Von 1900 bis 2006 verfolgt die Erzählung das Leben der georgischen Familie Jaschi entlang der großen historischen Zäsuren der Entstehung und des Niedergangs der Sowjetunion. Chronologisch schildern die sieben, jeweils einem Familienmitglied zugeordneten Bücher die Verflechtungen von Familien- und Weltgeschichte - denn es sind besonders die Männer, die sich auf die sowjetische Politik als Offiziere, Apparatschiks oder Widerstandskämpfer einlassen, und damit Leid über alle Jaschis bringen. Erzählt wird der Roman von Niza Jaschi, die der vierten vorgestellten Generation der Familie angehört und zunächst als Erzählerin wider Willen agiert: Bereits in den 1990er Jahren hat sie die meisten Verbindungen zu ihrer Familie in Georgien gekappt, um in Deutschland ein neues Leben zu finden. Zum Erzählen bringt sie erst ihre Nichte Brilka, die sie 2006 kennenlernt, als die Zwölfjährige während einer Europatournee ihrer Tanztruppe ausreißt und von der in Berlin lebenden Tante wieder eingefangen werden muss. Um ihr die lückenhaft überlieferte Familiengeschichte näherzubringen, beginnt Niza ihre Recherche nach den sieben Leben der Jaschis, auf dass Brilka ein selbstbestimmtes, von der Vergangenheit unabhängiges achtes Leben führen kann. 
In diesem kurzen Handlungsabriss deuten sich bereits die motivischen Bezüge an, die eine Kontextualisierung des Romans der gebürtigen Georgierin innerhalb des Felds der deutschsprachigen Gegenwartsliteratur erlauben. So lässt sich HARATISCHWILIS Schreiben zunächst mit verschiedenen anderen literarischen Texten in Verbindung bringen, die seit der Jahrtausendwende die Lebenswelten ehemals sozialistischer Länder ins Zentrum ihres Erzählens stellen (vgl. EIGLER 2010:11; LÖFFLER 2003; ROHRWASSER 2003). Diese Texte erfüllen nach ŠLIBAR (2016:23) eine „Funktion der Wissensvermittlung, des Informierens über Regionen und Kulturen, die aus westlicher Sicht hinter dem Eisernen Vorhang verschwunden waren“, was - nebenbei bemerkt - nicht zuletzt auch auf „die interkulturelle Leistungsfähigkeit des Familiengenres“ hindeutet. Doch das Erzählen im Achten Leben ist nicht nur ein Erzählen von einer in Deutschland eher unbekannten ehemaligen Sowjetrepublik. Es ist weiterhin auch nach Autorin, Erzählerin und fokussierter Familiengeschichte ein spezifisch weiblich markiertes Erzählen und steht so in einer Reihe mit verschiedenen anderen zeitgenössischen Romanen, die sich bemühen, die lange vorwiegend männlich konnotierte Vorstellung von Generationenabfolgen, wie sie beispielsweise KARL MANNHEIM (1928 / $\left.{ }^{2} 1970\right)$ konzeptualisierte, aufzubrechen (vgl. SILLER 2016:41-44). ${ }^{1}$

Diese Konzentration des Erzählens auf die Generation als „Nahtstelle von individueller und kollektiver Geschichte“ (EIGLER 2005:10) ist es auch, die Das achte Leben jenseits der bereits vorgestellten Motivbezüge gattungspoetisch als einen der Erzähltexte definiert, die seit der Jahrtausendwende die deutschsprachige Gegenwartsliteratur als Familien- oder Generationenromane stark prägen. ${ }^{2}$ Kennzeichnend für diese Romangattung, hinsichtlich deren begrifflicher Binnendifferenzierung in der Forschung noch immer kein Konsens erreicht

1 Einen exemplarischen Überblick über auf weibliche Familienmitglieder fokussierende Generationenromane bietet ŠLIBAR (2016). Auf die Kategorie des Geschlechts konzentrierte Einzelanalysen liegen beispielsweise für Helene Flöss' Mütterlicherseits von 2010 (vgl. SILlER 2016) und Sabine Scholls Wir sind die Früchte des Zorns von 2013 (vgl. SILLER 2016; SCHÜLKE 2016) vor.

2 Die zu dieser Romangattung inzwischen vorliegende Forschung ist so umfangreich, dass hier nur auf einige Titel verwiesen werden kann: Den Beginn der wissenschaftlichen Auseinandersetzung mit entsprechenden Texten markiert der Aufsatz des Soziologen HARALD WELZER (2004), der für die Jahre nach der Jahrtausendwende eine „Konjunktur der Familien- und Generationenromane“ feststellt - ein Befund, welcher schnell $\mathrm{zu}$ einer breiten auch literaturwissenschaftlichen Rezeption, beispielsweise durch BERNHARD JAHN (2005), führte. Die erste umfangreichere 
wurde ${ }^{3}$ ist eine narrative Verbindung von Familien- und Zeitgeschichte, die im erzählten Leben der Familienmitglieder die Historie aufscheinen lässt. Neben ihrer Analyse unter Rückgriff auf das Generationenparadigma, wie es prominent in Arbeiten von ALEIDA ASSMANN (2007:70-95) und besonders SigRID WEIGEL (2006) zum Ausdruck kommt, wurde diese Verbindung von Historie und Familiengeschichte(n) in der literaturwissenschaftlichen Forschung von Beginn an vorwiegend hinsichtlich der Frage untersucht, wie in der narrativen Inszenierung der Spannung zwischen Individual- und Weltgeschichte Gedächtnis- und Erinnerungsprozesse literarisch bearbeitet werden (EIGLER 2005). ${ }^{4}$

Was in dieser auf Gedächtnis- und Erinnerungsprozesse fokussierenden Rezeption der Generationenromane in den Blick gerät, sind weniger stabile Identitäten und historische Kontinuitäten, sondern eher historische „Brüche und Leerstellen“ (EIGLER 2005:11), sind „Lücken und Missstände, verfehlte Chancen“(GISBERTZ

Untersuchung von Familien- und Generationenromanen, die ihren Fokus auf die narrative Bearbeitung von Themenkomplexen um Gedächtnis und Geschichte legt, bietet FRIEDERIKE EIGLER (2005), in den letzten Jahren ergänzt um JULIAN REIDYS (2013) Überlegungen zum postheroischen Generationenroman und den Lektüren ANNA-KATHARINA GiSBERTZ' (2018), die entsprechende Texte vor allem als Zeitromane liest. Eine kurze Begriffs- und Gattungsgeschichte des Familien- bzw. Generationenromans bieten GALLI / COSTAGLI (2010:7-18), ein exemplarischer Überblick über gattungsbildende Beispiele seit der Antike findet sich bei GisBERTZ (2018:38-58).

Die ältere Bezeichnung ,Familienroman' wird in der deutschsprachigen Forschung inzwischen häufig, nicht zuletzt wegen ihrer psychoanalytischen Besetzung durch FREUDS Der Familienroman der Neurotiker (1909/ $/ 1970)$, zu Gunsten des Generationenroman-Begriffs vermieden, die beiden Termini finden sich aber auch noch parallel verwendet. Versuche einer systematischen Unterscheidung zwischen Familien- und Generationenroman, wie sie GALLI / CoSTAGLI (2010:8f.) vornehmen, nach der erster ,als allgemeine Bezeichnung für Texte mit Handlungsfokus innerhalb einer Familie gelten [kann], während der zweite Romane betrifft, die chronologisch mehrere Generationen umfassen“, werden zwar durchaus aufgegriffen, haben sich aber noch nicht allgemein durchgesetzt. Da es im Achten Leben besonders der Begriff der Generation ist, der nach CosTAGLi (2010:163) die entscheidende Nahtstelle zwischen Familien- und Zeitgeschichte durch seine doppelte Anwendbarkeit - Generationen gliedern sowohl historische Geschichtsverläufe als auch (fiktionale) Familienverbünde - markiert, spreche ich im Folgenden unter Vermeidung der unbefriedigenden Doppelformel ,Familien- und Generationenroman' von HARATISCHWILIS Text als einem Generationenroman. in zeitgenössischen österreichischen Generationenromanen nach. 
2018:14f.), die für die Erzählgegenwart sinnstiftend aktualisiert - das heißt: narrativiert und geordnet - werden wollen.

\section{Bruch und Naht: Erzählung und Erzählinstanz}

Auch HARATISCHWILIS Das achte Leben kann unter dem strukturellen wie inhaltlichen Paradigma des Bruches gelesen werden, der sich hier nicht nur in einer brüchigen und zunächst unverfügbaren Familiengeschichte manifestiert, sondern auch die Erzählinstanz selbst betrifft. Denn Niza ist nicht nur die Erzählerin der Geschichten der Jaschis, sondern auch selbst ein Teil der generationalen Familienabfolge, die sie in ihrer Erzählung rekonstruiert. GISBERTZ (2018:115) bemerkt zu Recht, dass dieser Bruch, der eine Verdoppelung der Figurenrolle zur Folge hat und Niza damit eine Distanzierung von erfahrenen Traumata ermöglicht, die Voraussetzung für ihre Rolle als Erzählerin ist. Es wird aber zu zeigen sein, dass der hier angelegte Bruch, der im Übrigen typisch für personale Erzählinstanzen in Generationenromanen ist, problematische Folgen für den Akt des Erzählens selbst nach sich zieht. Die eingangs als typisch für Generationenromane bestimmte und von der Erzählinstanz zu gestaltende ,generationale Nahtstelle' ist damit im Achten Leben eine doppelte: Es ist zum einen die Nahtstelle zwischen Familien- und Zeitgeschichte, aus der nach GEIER (2014:131) potenzielle Spannungen und Konkurrenzen ,zwischen ,Familiengedächtnis ' und ,kollektivem Gedächtnis ““ erwachsen. ${ }^{5}$ Zum anderen verläuft die zweite Nahtstelle direkt durch Niza selbst, die gleichzeitig erzählte Figur und Erzählinstanz ist - wie sie sich auch Brilka zunächst als metanarratives Du und Adressatin ihrer Erzählung konstruiert. Doch zum Ende des Romans erzählt Niza von ihrer Nichte zusätzlich als Figur in der generationalen Familienabfolge, was Brilka ebenfalls eine doppelte Funktion in der Narration einräumt. ${ }^{6}$

Dass aus einer narrativen Privilegierung subjektiven Erinnerns gegenüber der kollektiven und dokumentarisch-methodisch abgesicherten Geschichtsschreibung neben Chancen auch Gefahren erwachsen, zeigt ANDREA GEIER (2014) am Beispiel von Uwe Timms Am Beispiel meines Bruders von 2003 und insbesondere Ulla Hahns Unscharfe Bilder aus dem gleichen Jahr, die sich an der Bewertung und Rekonstruktion familiärer Verstrickungen im Nationalsozialismus abarbeiten.

Auch die Problematik dieser zweiten ,Nahtstelle' kann dabei durchaus als symptomatisch für Generationenromane angesehen werden, identifiziert doch schon EIGLER (2005:10) bei der Analyse ihres Korpus als eine interpretatorische Hauptfrage, ,auf welche Weise sich die Erzählfiguren als Teil dieser rekonstruierten Geschichten darstellen“. 
Diese durch Niza selbst verlaufende Nahtstelle, die ihre Spaltung zwischen den Rollen von Figur und Erzählinstanz markiert, ist es, die den Akt des Erzählens für sie so erschwert. Während Niza einerseits in einfühlender Manier die Lebensgeschichte(n) der einzelnen Familienmitglieder erzählt und dabei organisiert, legt sie andererseits in zahlreichen metanarrativen Reflexionen und Dialogen Brilka als dem Du ihrer Erzählung Rechenschaft über Voraussetzungen, Methoden und Grenzen ihres Erzählens ab. Potenzielle Probleme, die einer Erzählinstanz erwachsen, welche einerseits nah an ihren Figuren erzählt, andererseits die Rolle der Erzählerin für sich selbst immer wieder hinterfragt, wurden für Das achte Leben bereits diskutiert. So sieht ROMAN HALFMANN (2017:17f.) den Roman als „durchaus gescheitert“ an, weil er zwischen zwei Möglichkeiten ,[n]ämlich eine postmoderne Erzählung mit zweifelhaftem Erzähler zu sein oder eben ein auf Echtheit basierender Roman" - changiere: Nizas Bemühung, während der Erzählung der Familiengeschichte ,ganz mit der [jeweiligen] Hauptperson zu verschmelzen“, die sich in ihrem „quasi-authentischen Ton“ ausdrücke, müsse nämlich scheitern, da sie als Erzählerin, die den Großteil ihrer Geschichte eben nicht erlebt hat, in postmoderner Manier ,immer wieder mit Sentenzen aus der Rolle" falle.

Dieses Urteil erscheint mir problematisch, insofern es die fiktionsinternen Brüche der Erzählpoetik Nizas als fiktionsexterne Schwäche der Erzählweise NINO HARATISCHWILIS ausweist. In der Folge wird zu zeigen sein, dass es vielmehr genau diese brüchige Erzählpoetik Nizas ist, durch die in Das achte Leben Möglichkeiten, vor allem aber Grenzen der sinnstiftenden narrativen Organisation von Zeitverläufen im Generationenroman reflektiert werden. Auflösbar sind die Widersprüche dieser Erzählpoetik, so die These der folgenden Überlegungen, letztlich nicht mehr im Medium der Schrift: Das Ende von HARATISCHWILIS Roman verweist die Lösung des Problems sinnstiftender Vergangenheitsdeutung nicht nur mit Brilka in die nächste Generation, sondern auch in die neue Medialität des Tanzes. Es ist dieser in Roman und Erzählpoetik angelegte Medienwechsel, der bereits die Adaption von HARATISCHWILIS Roman auf der Theaterbühne vorbereitet, für die JETTE STECKELS Uraufführungsinszenierung am Thalia Theater Hamburg (2017) das erste Beispiel ist.

\section{Teppich und Faden: Textile Erzählpoetik}

Nizas Erzählpoetik findet sich schon im Prolog des Romans im wahrsten Wortsinn ausgebreitet: Als sie ungefähr neun Jahre alt ist, beobachtet die kleine Niza ihre Urgroßmutter Stasia, wie sie einen granatapfelroten ramponierten Teppich 
ausklopft und zur Restauration vorbereitet. Was in dem sich entspinnenden Gespräch Stasias mit ihrer Urenkelin neben der poetologischen Bedeutung der Teppich-Episode, um die es in der Folge vor allem gehen soll, aufscheint, ist das Motiv der matrilinear weitergegebenen textilen Handwerkskunst, das auch in anderen Generationenromanen, die auf eine weibliche Generationenfolge fokussieren, bearbeitet wird: ${ }^{7}$ Wie dort stiftet damit Stoff auch im Achten Leben eine „transgenerationelle[ ] Verbindung zwischen den Frauenfiguren“" (SILLER 2016:52).

„[D]ie Geschichte des Teppichs“ wird von Niza in ihrer Erzählgegenwart als entscheidend für ihre weitere Entwicklung eingeschätzt, insofern Stasias Erläuterungen zum Stoff und seiner Erneuerung sie „,vielleicht am nachhaltigsten geprägt [haben]“ (HARATISCHWILI 2014:29). Die Wichtigkeit der Szene auch für die Erzählerin Niza wird damit schon zu Beginn des Romans deutlich markiert - eine Wichtigkeit, die sich im Verlauf des Textes durch das häufige Auftauchen von auf den Teppich rekurrierenden Vergleichen und Metaphern in Nizas metanarrativen Überlegungen bestätigt (z.B. HARATISCHWILI 2014:143, 212, 1198, 1255).

Die Leser*innen erfahren von besagtem Teppich, dass er bereits Stasias Großmutter gehörte, was die erzählte Zeit und Generationenfolge noch weiter in eine Vergangenheit vor 1900 öffnet. Da er aber von Stasia und ihrer Schwester Christine nicht wertgeschätzt und gepflegt wurde, befindet er sich nun in dem schlechten Zustand, der eine Restauration erfordert. Auf die Bemerkung der kleinen Niza, man könne doch Altes nicht neu machen, antwortet Stasia:

Natürlich kann man das. Das Alte wird neu, also anders sein, nie mehr genau das, was es einmal war, das ist auch nicht der Sinn der Sache. Es ist besser und interessanter, wenn sich etwas verwandelt. Wir machen ihn neu, hängen ihn auf und sehen, was passiert. (HARATISCHWILI 2014:30)

Bereits hier wird also die Restauration als transformierender Prozess greifbar, dem aber noch eine genauere Zweckbestimmung fehlt. Dies merkt auch Niza mit der Frage an, was denn eigentlich der Sinn des mühsamen Unterfangens sei. Die Antwort Stasias erscheint entscheidend für die Poetik des Erzählens im Roman, weshalb ich sie hier ausführlich zitiere:

Ein Teppich ist eine Geschichte. In ihr verbergen sich wiederum unzählige andere Geschichten. [...] Das sind die einzelnen Fäden. Der einzelne Faden ist wiederum eine einzelne Geschichte, verstehst du mich? [...] Du bist ein Faden, ich bin ein

7 So geht SiLLER (2016) den um „Wolle, Stoffe und Kleider“ (SILLER 2016:50) kreisenden Erzählsträngen in Wir sind die Früchte des Zorns und Mütterlicherseits nach (vgl. SILLER 2016:50-53); für letzteren Roman analysiert den Motivkomplex auch SCHÜLKE (2016:127f.). 
Faden, zusammen ergeben wir eine kleine Verzierung, mit vielen anderen Fäden zusammen ergeben wir ein Muster. [...] Die Muster sind einzeln schwer zugänglich, aber wenn man sie im Zusammenhang betrachtet, dann erschließen sich einem viele fantastische Dinge. [...] Teppiche sind aus Geschichten gewoben. Also muss man sie wahren und pflegen. (HARATISCHWILI 2014:30)

Was Stasia hier aus der materiellen Beschreibung des Textils entwickelt, ist ein Bild des Geschichte(n)-Erzählens als Verknüpfungsleistung, das in dreifacher Hinsicht für Nizas Erzählpoetik verbindlich wird.

Typisch für den Generationenroman erscheint im Bild des Teppichs Erzählen, erstens, als Zeit- und Familiengeschichte verflechtendes Erzählen. Das Grundgestaltungsmerkmal des zu restaurierenden Textils ist das Zusammenspiel zwischen den „granatroten Farbtönen“ (HARATISCHWILI 2014:29) der Grundierung und den farbigen Fäden, sodass sich „,bunte[ ] Ornamente auf der roten Fläche“ (HARATISCHWILI 2014:30) ergeben. Ohne die rote Grundfläche fehlt den Mustern der Hintergrund, vor dem sie erst wahrgenommen werden können, andererseits ist ohne die von Stasia als „einfach fabelhaft“ (HARATISCHWILI 2014:30) bezeichneten Muster die rote Grundfläche des Teppichs unstrukturiert und damit inkommensurabel. Was Niza im Akt ihres Erzählens also sinnstiftend aufeinander beziehen muss, ist das Leben ihrer Familie und das historische „rote[ ] Jahrhundert“ (HARATISCHWILI 2014:16) der Sowjetunion. Nicht nur HARATISCHWILIS Generationenroman, sondern auch Nizas Narrationsprojekt wird damit durch den von CostagLI (2010:160) beschriebenen Chronotopos der Generation strukturiert, in dem ,die Einzelschicksale der Protagonisten durch das permanente Zitieren zeitgeschichtlicher Ereignisse in den , Weltlauf eingebettet werden".

Das Projekt der Restauration des Teppichs markiert das Erzählen im Roman, zweitens, als vorlagenabhängiges und-aktualisierendes Erzählen. Denn Stasias Ziel ist es keinesfalls, einen neuen Teppich zu knüpfen. Vielmehr liegt das Textil - wie zerschlissen auch immer - bereits vor. Nizas Erzählen wird so als WiederErzählen einer Geschichte und damit als vorlagenabhängiges Erzählen markiert. Doch diese Abhängigkeit erscheint keinesfalls einseitig: Der Teppich ist deshalb restaurationsbedürftig, weil er zu lange ignoriert wurde. Dies heißt für das Erzählen, dass es zwar als Wieder-Erzählen einer Vorlage bedarf, andererseits aber auch diese Vorlage auf eine ständige erzählerische Aktualisierung angewiesen ist. Was dabei in seiner Wichtigkeit markiert wird, ist eher das Erzählen als die Erzählung, ist eher die Narration als das Narrativ. Dazu passt, dass Niza an anderer Stelle im Roman gerade Stasia, also die Person, die die Restauration des Teppichs vorbereitet, als große Geschichtenerzählerin inszeniert: 
Die sonst so wortkarge Stasia verwandelte sich in Scheherazade und führte mich in den unglaublichsten Farben in eine verborgene Welt ein. Mit ihrer blumigen Sprache, mit dramatischen Höhepunkten und spannungsvollen Wendungen, in verteilten Rollen und mit verstellter Stimme ließ sie Vergangenes wieder zur Gegenwart werden. (HARATISCHWILI 2014:870)

Im Vergleich mit Scheherazade wird Stasias Erzählen in eine Tradition eingereiht, deren dominantes Merkmal ihre Mündlichkeit ist. Gerade in der Performativität dieser Erzählung als mündlicher Erzählung liegt ihre Kraft begründet, die die Vergangenheit reaktualisiert.

Doch trotz seiner Vorlagenabhängigkeit wird das Erzählen im Bild der Teppichrestauration, drittens, auch als subjektives Erzählen bestimmt. Denn das Ergebnis des von Stasia angestoßenen textilen Restaurationsprozesses erscheint völlig unvorhersehbar, Nizas Urgroßmutter will den fertigen Teppich aufhängen und „sehen, was passiert“ (HARATISCHWILI 2014:30). Dies bedeutet, dass die aktualisierende Narration zwar auf bekannten Narrativen beruht, diese aber neu bearbeitet und verknüpft und dadurch eine eigenständige Sinnstiftung vornimmt. Niza tritt dann auch in der Folge als Erzählerin auf, deren restaurierendes Erzählen Vergangenheit nicht so sehr konserviert, sondern aus der Perspektive der eigenen Gegenwart - und das heißt vor allem: für Brilka - neu zu erzählen versucht. Dieses Neu-Erzählen ist schon deshalb eine Notwendigkeit, weil Niza den Großteil der von ihr referierten Familiengeschichte nicht erlebt hat, sondern erst spät, ,in unsere Geschichte hineingeboren“ wurde, sodass sie sich nicht auf eigene Erinnerungen verlassen kann, sondern sich ,mit fremden begnügen [muss], die sich dann mit meiner Vorstellung kreuzen" (HARATISCHWILI 2014:682). Gleichzeitig ist sich Niza aber über diese generationell bedingte Subjektivität ihres Erzählens hinaus auch über die Selektivität jeder Art von Erinnerung und damit Geschichtsschreibung bewusst:

Wir entscheiden uns dafür, an was wir uns erinnern wollen und an was nicht. Die Zeit hat damit nichts zu tun. Der Zeit ist das egal. Aber an unserer Geschichte ist es das Ungerechte, Brilka, das weder mir noch dir die Möglichkeit gegeben ist, an alles erinnern zu können, eben auch an das Vergessene, dass auch ich - für dich auswählen muss, entscheiden, was erzählenswichtig ist und was nicht; eine bisweilen unmögliche Aufgabe, scheint mir. (HARATISCHWILI 2014:522)

Erzählen als ein vorhandene Erinnerungen reaktualisierender und damit subjektiver Prozess, der Familien- und Zeitgeschichte nicht nur verknüpfen, sondern sinnstiftend aufeinander beziehen soll - in dieser in die Teppichmetapher eingelagerten Poetik scheint bereits das Hauptproblem von Nizas Erzählprojekt auf, das sich abermals in der Materialität der Teppichmetapher gefasst findet und als Versuch des Erzählens zwischen Faden und Draufsicht expliziert werden kann. 


\section{Zwischen Faden und Draufsicht: Nizas doppelt motivierter Erzählprozess}

Im Achten Leben ist Niza nicht nur Restauratorin des Teppichs der Familienund Zeitgeschichte, ihr Leben ist nach Stasia selbst ein Faden im Teppich. So findet sich die einführend bereits als typisch für den Generationenroman skizzierte Doppelrolle verbildlicht, die das Erzählen für Niza im Roman so schmerzvoll und zu einem fast unmöglichen Unterfangen machen wird: Denn Stasia weist darauf hin, dass vom einzelnen Faden ausgehend keine Aussage über die Muster getroffen werden kann, zu denen sich die verschiedenen Fäden verbinden. Erst die Draufsicht auf den gesamten Teppich bietet die Perspektive, aus der sich der Verlauf der Fäden vor dem roten Hintergrund als Muster interpretieren lässt (vgl. HARATISCHWILI 2014:30). Die Hauptschwierigkeit des Erzählens für Niza ist damit die Tatsache, dass dieses wegen ihrer doppelten Rolle - Restauratorin und Faden - doppelt motiviert ist: Zum einen will sie für Brilka die Familiengeschichte insgesamt sinnstiftend darstellen, zum anderen versucht sie aber auch zunehmend verzweifelt das Muster zu erkennen, das ihr eigener Faden im Gewebe bildet.

Ihr doppeltes erzählerisches Scheitern, das sich aus dieser doppelten Motivation ergibt, wird Niza besonders in einer Szene des Romans vor Augen geführt, in der sie auf ihrer Recherchereise Giorgi Alani, einen alten Freund ihres Großvaters, aufsucht, von dem sie sich Auskünfte über die Teile der Familienhistorie und ihrer Verbindung zur Geschichte der Sowjetunion erhofft, die ihr noch unklar sind. Doch im Gespräch mit dem ihr bereitwillig Auskunft gebenden Ex-Geheimdienstler bemerkt Niza, dass sie die hier offenbarten Informationen weder bewältigen noch sinnvoll in ihre Erzählung integrieren kann: „In meinem Kopf versammelten sich alle Gespenster, und er webte den Teppich vor meinen Augen weiter. Ich sah ihm dabei zu, immer noch unfähig, meinen Faden in all diesen verwirrenden Mustern zu erkennen“ (HARATISCHWILI 2014:1198). In diesem wieder auf die Teppichmetapher rekurrierenden Bild ist es Alani, der die aktive Position einnimmt und als Erzähler den Teppich der Geschichte ,weiterwebt', während Niza in Passivität verharrt und ihren eigenen Faden im Gespinst der sich vor ihr entfaltenden Geschichte nicht erkennen kann. In dieser Situation nimmt sie zur Strategie der Spaltung Zuflucht: Sie bittet Alani, ihr nur die historische Geschichte der Sowjetunion, nicht ihre persönliche Familiengeschichte zu erzählen. Doch ihr Gesprächspartner glaubt nicht an die Möglichkeit dieser Aufspaltung: „Sie sollten Ihre Geschichte nicht von der allgemeinen trennen, sich nicht von sich selbst zu amputieren versuchen“ 
(HARATISCHWILI 2014:1200). Im Vergleich der Trennung von eigener und Zeitgeschichte mit einer Selbstamputation wird Niza somit endgültig die Ausweglosigkeit des Widerspruchs aufgezeigt, in den sie die ,textile Poetik' geführt hat, an der sie seit der Teppichrestauration Stasias ihr Erzählen ausrichtet.

Je deutlicher sich Niza der Defizite ihrer narrativen Organisationbemühungen bewusst wird, desto mehr versucht sie mit ihrer Erzählung stattdessen, die generationale Strukturierungsmacht der Zeit selbst zu überwinden. Dieses Vorhaben manifestiert sich in dem Wunsch, durch ihr Erzählen Brilka, deren selbstgewählter Name - eigentlich heißt sie Anastasia - im Georgischen keine Bedeutung hat und damit „unbeschriftet und unstigmatisiert“ (HARATISCHWILI 2014:15) ist, die Chance eines Neuanfangs jenseits der in den ersten sieben Büchern des Romans entworfenen Leben der Jaschis zu ermöglichen. In diesem Sinne erkennt Niza in impliziter Anlehnung an Franz Kafka ${ }^{8}$ als ihre Aufgabe ,die Pflicht einer Axt, die die Zeiten zerschmettert, für dich“ (HARATISCHWILI 2014:523). So gibt sie ihrer Nichte schon im Prolog den Auftrag: „Durchbrich diese Geschichte und lass sie hinter dir" (HARATISCHWILI 2014:17). Ziel des gesamten Narrativs ist damit nicht mehr eine sinnstiftende Verknüpfung der Familien- und Zeitgeschichte, sondern deren Auflösung. Zum Fixpukt von Nizas Erzählen wird die Einführung Brilkas

[i]n diese Geschichte hier, die nur erzählt werden musste, um zu dir zu gelangen. $\mathrm{Zu}$ dir und somit zum Anfang. In diese Geschichte, die nur deswegen geschrieben werden musste, damit du noch einmal auf die Welt kommen und eine Möglichkeit bekommen konntest, alles neu und alles anders zu beginnen. (HARATISCHWILI 2014:1135)

\section{Literatur und Tanz: Medienwechsel}

1993 endet nicht nur durch den Eintritt Georgiens in die Gemeinschaft Unabhängiger Staaten und den endgültigen faktischen Verlust Abchasiens das sowjetische Jahrhundert des Landes, sondern mit Brilkas Geburt auch die bisherige

8 So schreibt KAFKA in seinem Brief an Oskar Pollak am 27.01.1904: ,[E]in Buch muß die Axt sein für das gefrorene Meer in uns" (1904 / 1958:28). In der russischen Literaturgeschichte wird die Axt als Mordinstrument insbesondere in Fjodor Michailowitsch Dostojewskis Schuld und Sühne motivisch; in Vladimir Sorokins Roman Roman wird mit ihrer Hilfe gar die ganze auf einer Hochzeit versammelte Verwandtschaft niedergemetzelt und damit nicht nur die Strukturierungsmacht des Familienverbundes zerstört, sondern in der immer weiter eskalierenden literarischen Gewaltorgie jeder Form von verbindlicher Ordnung - auch des Romans als Gattung - eine Absage erteilt. (Für den Hinweis auf das Motiv der Axt in der russischen Literatur danke ich Gudrun Heidemann.) 
Familiengeschichte der Jaschis, die vor allem durch die Lebenswege ihrer männlichen Mitglieder so eng mit der sowjetischen Geschichte verbunden war. In diesem Umbruch, der in Nizas Erzählung genauso als familiärer wie historischer markiert wird, kommt aber die metaphorische Erzählpoetik des Teppichs mit ihrem Zusammenspiel von roter Grundierung der Zeitgeschichte und bunten Fäden der familiären Lebensläufe auch hinsichtlich der Bildlichkeit an ihre Grenzen. Der Teppich, der im Roman auch als ,metonymische Repräsentation von Zeitlichkeit und Geschichte" interpretiert werden kann, als die COSTAGLI (2010:168) in anderen Generationenromanen Objekte wie „Memoiren, Orte, Fotos“ ausmacht, die „eine die Zeit übergreifende Kontinuität dort wieder [geben], wo das Generationenschema fragmentiert erscheint", kann weder eine funktionale Erzählpoetik für Niza noch eine Familien- und Zeitgeschichte zusammenführende Historie stiften.

Mit dem Abschluss des Achten Lebens wird aber nicht nur die individuelle Erzählpoetik Nizas als dysfunktional markiert, sondern das generationale Erzählen als literarisches Erzählen selbst problematisiert. Was am Ende des Romans inszeniert wird, ist nicht nur ein letzter Generationen-, sondern auch ein Medienwechsel. Brilka erwartet von Niza nicht die sinnstiftende Fixierung der Familiengeschichte als Roman, wie am Ende des Buches - aber chronologisch am Anfang von Nizas Erzählbemühungen - aus Brilkas Tagebuchaufzeichnungen klar wird. In diesen formuliert die Nichte die Frage vor, die sie ihrer Tante stellen möchte: „Willst du für meine Choreographie die Geschichte schreiben?" (HARATISCHWILI 2014:1254) Worum es Brilka also geht, ist die Transformation der Generationenerzählung in das ephemere und performative Medium des Tanzes. Die Befreiung, die sich Niza für ihre Nichte wünscht, bedeutet damit gerade nicht eine Abkehr von der Familiengeschichte, sondern abermals deren Aktualisierung, diesmal jedoch in einem anderen Medium, das die Performanz des oralen Erzählvorgangs wieder aufnimmt. In diesem Neuansatz bei der Beschäftigung mit Generationengeschichte bricht Brilka mit Nizas Erzähltradition. Gleichzeitig schreibt sie sich aber auch in eine Traditionslinie der Familienhistorie ein, zu der ihre Tante nie einen Zugang gefunden hat.

Denn das Muster der performativen Verbindung von Erzählung, Musik und Tanz beginnt nicht bei Brilka, sondern durchzieht den Roman schon zuvor: Die Musik, die Brilka neben den Texten ihrer Tante für ihr Projekt verwenden will, stammt von ihrer Urgroßtante Kitty, die aus der Sowjetunion fliehen musste und in Großbritannien Karriere als Sängerin machte. Ihre Affinität zur Musik mag dabei genealogisch in der Begeisterung begründet liegen, die Kittys Mutter Stasia dem Tanz entgegenbringt: Ihr Kindheitstraum war es, Mitglied der Ballets 
Russes zu werden, noch im hohen Alter gibt sie Kindern Ballettunterricht. Das versucht sie auch bei ihrer Urenkelin Niza, doch diese findet zur Musik keinen Zugang, sondern konzentriert sich nur auf das Erzählen der Urgroßmutter (vgl. HARATISCHWILI 2014:870-872). So kommt in Brilka ein den Roman durchziehendes matrilineares Muster zur Erfüllung: Tänzerin wie ihre Ururgroßmutter Stasia und musikaffin wie ihre Urgroßtante Kitty unternimmt sie eine neue Aktualisierung der Generationenerzählung in der Sphäre von Musik, Tanz und Körperlichkeit. Ihre Tante Niza kann ihr auf diesem Weg nicht folgen, sondern ihn nur vorbereiten. Die letzte nummerierte Seite des Romans, die das „Buch Brilka“ andeutet, bleibt leer (vgl. HARATISCHWILI 2014:1279). Wie GiSBERTZ (2018:125) mit Recht anmerkt, liegt dem Roman - und damit auch Nizas Erzählen - letztlich eine „Einsicht in die Unzuverlässigkeit der Sprache“ zu Grunde, ,in der sich Vergangenes nicht vollständig erschließt“: Die Generationenerzählung als Roman bricht ab, als verkörperte, theatrale Inszenierung wird sie in ein neues Medium überführt.

\section{Theatrale Adaption: JeTte STECKELS Inszenierung am Thalia Theater Hamburg}

Es ist diese von Brilka geplante Transformation der Generationenerzählung vom Medium der Schrift in die theatrale Verkörperung, die für JETTE STECKELS Adaption ${ }^{9}$ des Romans am Thalia Theater Hamburg den Rahmen bietet, welche am 8. April 2017 uraufgeführt wurde. ${ }^{10}$ Entsprechend spielt in der fast fünfstündigen Inszenierung der Tanz eine entscheidende Rolle: Bei STECKEL entfaltet sich die Familien- und Zeitgeschichte auf der Bühne auch als Tanzgeschichte vom russischen Ballett über Soldatentänze der Roten Armee bis zu den Paartänzen der sowjetischen Elite nach dem Krieg.

Doch ist der Theaterabend nicht reines Tanztheater auf leerer Bühne: Das Narrativ von der Restauration des Teppichs, das für den Roman als Bild für Nizas Erzählpoetik ausgelegt wurde, wird ebenfalls theatral umgesetzt, jedoch findet sich dieser rote Teppich bei STECKEL anders inszeniert: Er ist hier das aus dem Schnürboden senkrecht herabhängende, alles dominierende Bühnenelement

$9 \quad$ Die Bühnenfassung erarbeiteten die Dramaturginnen Emilia Heinrich und Julia Lochte gemeinsam mit der Regisseurin.

10 Die Grundlage der folgenden Inszenierungsanalyse bilden ein Aufführungsbesuch im März 2018 sowie ein mir freundlicherweise vom Thalia Theater zur Verfügung gestellter Aufführungsmitschnitt. 
(Bühne: Florian Lösche), das im Verlauf des Abends immer weiter abgerollt wird und verschiedene Funktionen übernimmt. Dabei dient das Textil beispielsweise als Projektionsfläche für historisches Videomaterial, wie es auch immer wieder als Symbol für die Konfrontation der Figuren mit der Zeitgeschichte funktionalisiert wird. Denn der große Unterschied zum Roman besteht darin, dass dieser rote Teppich in der Inszenierung ausschließlich die Zeitgeschichte repräsentiert, während sich die individuellen Geschichten der Familienmitglieder vor ihm, hinter ihm und gegen ihn entfalten. So wirft der von Sebastian Rudolph gespielte mächtige sowjetische Funktionär Kostja Jaschi einen Widersacher, den er durch Ausnutzung der ihm qua Parteiamt zur Verfügung stehenden Machtmittel außer Gefecht setzt, immer wieder in den Teppich, bis sein Gegner entkräftet liegen bleibt. Als dagegen Kostja selbst von der neuen Machtelite kaltgestellt wird, findet die Inszenierung dafür das Bild des gebrochenen Parteisoldaten, der vergeblich versucht, am Teppich nach oben zu klettern, dabei aber an dessen Oberfläche abgleitet. Die Muster, die diesen Teppich durchziehen, werden dementsprechend nicht durch die Lebens-Fäden der Familie Jaschi bestimmt, sondern von sowjetischer Imagologie, von Ähren, Hammer und Sichel, Waffen - und den Konterfeis der Parteiführer und Staatslenker von Lenin bis Putin.

Repräsentiert der Teppich also die Zeitgeschichte, findet die Inszenierung für die private Familiengeschichte einen anderen Raum: Der Teppich lässt sich nämlich auch um die eigene Achse drehen, sodass sich immer wieder ein Bühnenraum ,hinter der sowjetischen Geschichte' ergibt. Dieser ist bestimmt durch den topischen Familientisch, an dem die Verhandlungen, Streitigkeiten und Handlungsplanungen stattfinden, mit denen die Jaschis versuchen, sich gegenüber der sowjetischen Historie zu behaupten. Doch die räumliche Bühnensituation macht auch deutlich, dass es vor dieser Zeitgeschichte letztlich kein Entkommen gibt. Auch der scheinbar private Familienraum wird visuell durch die Rückseite des Teppichs dominiert und die erbarmungslose zirkuläre Bewegung der Drehbühne, die sich in den oftmals konzentrisch-kreisenden Gängen der Darstellenden spiegelt, scheucht die Familienmitglieder immer wieder vor die Teppichvorderseite. Diese starke Identifikation des Teppichs mit dem, roten sowjetischen Jahrhundert' führt dazu, dass er in der Inszenierung folgerichtig in den Revolutionen des Jahres 1989 aus seiner Verankerung auf die Bühne fällt. In dem effektvollen Bild kommt die Geschichte der Sowjetunion an ein Ende, deren Ablaufen sich bereits über den ganzen Abend durch das ständig weiter fortschreitende Abrollen des Teppichs ankündigte.

Im Roman war der Teppich Bild für die enge Verknüpfung von Zeit- und Familiengeschichte genauso wie für die unmögliche Aufgabe von Nizas Erzählen, sowohl 
den Faden ihres eigenen Lebens zu entwirren als auch den sinnvollen Gesamtzusammenhang zwischen familiärer und politischer Generationengeschichte herzustellen. In der theatralen Inszenierung dagegen steht der Teppich für die gewaltvolle Zeitgeschichte, an der sich die Jaschis abarbeiten, ohne doch in ihren kreisenden Bewegungen von ihr loszukommen. Folgerichtig muss der Teppich als konkretes Bühnenelement am Ende der Inszenierung genauso verschwinden, wie er auch am Ende des Romans als Metapher für Nizas Erzählpoetik zur Auflösung gebracht wurde. Diese Entsorgung übernimmt die von Mirco Kreibich gespielte Brilka, die den Teppich in den Orchestergraben schiebt. Somit ist es nicht mehr Nizas Erzählung, die als Axt Zeit und Zeitgeschichte für Brilka zerschlagen muss, in STECKELS Inszenierung schafft sich Brilka selbst den Bühnenraum, den sie für ihren abschließenden Tanz benötigt. Die von Lisa Hagmeister gespielte Niza ist als Erzählinstanz so gut wie ausgeschaltet, lediglich zu Beginn und am Ende darf sie den Rahmen der Generationennarration liefern, die ansonsten nicht im engeren Sinne narrativ entfaltet, sondern theatral verkörpert wird.

Diese konzeptionelle Änderung ist zum einen Folge der medialen Transformation vom Buch auf die Bühne. Aber in Zeiten, in denen bei vergleichbaren Romanadaptionen oftmals durchgängig stabile Erzähler*innenfiguren den theatralen Handlungsverlauf organisieren, kommentieren und distanzieren, erscheint es dennoch angebracht, auf die genuin theatralen Mittel hinzuweisen, mit denen STECKEL diese Adaption bewerkstelligt. Ihr Schlüssel zur Organisation des Bühnengeschehens ist dabei Brilka. Schon die Tatsache, dass sie mit Mirco Kreibich von einem Mann gespielt wird, deutet auf das Transformations- und Verbindungspotenzial der Figur hin. Während in der Erzählpoetik des Romans der fein gewebte Teppich für die strukturierende Verflechtung der einzelnen Figurengeschichten zur Familiengeschichte sowie der Familien- und Zeitgeschichte zur Generationenerzählung steht, leistet in der Inszenierung Brilka diese Verflechtung. Denn Mirco Kreibich als Brilka übernimmt im Stück so gut wie jede Nebenrolle, spielt die Liebhaberinnen und Liebhaber der Jaschis genauso wie Folterknechte oder Familienfreunde. In verschiedensten Verkleidungen und Spielweisen taucht Brilka damit immer wieder in der Generationenerzählung auf, mal weiblich, mal männlich, mal Feind, mal Freund der Familie. Nie sind die Rollen - etwa durch ein bestimmtes Kostümteil oder eine Spielweise - aufeinander bezogen, immer lässt Brilka sich ganz auf die jeweilige Rolle ein, nie bemüht sie sich um eine kohärenzherstellende Spielweise, wo angesichts der chaotischen Zeitabläufe eben kein szenenübergreifender Sinnzusammenhang zu stiften ist. Dem Erzählen als Sinndeuten setzt Brilka so die vollständige Ver-Körperung, das Eintauchen in Rolle und Zeitablauf entgegen. 
Ihren Höhepunkt findet diese Ver-Körperung von Geschichte in Brilkas letztem Tanz am Ende des Stücks: Hier integriert sie nicht nur verschiedene Tanzstile von Ballett bis zum Ausdruckstanz, sondern ihre Gesten erinnern auch an Spielweisen der anderen Darstellenden. In Brilkas Tanz hat die Generationenerzählung eine Form gefunden, in der sie erinnert und transportiert werden kann, ohne in feststehenden Sinnstrukturen fixiert zu werden. Das, was sich dem Erzählen als Teppichrestaurieren widersetzte, kann der Tanz also performativ in Erscheinung treten lassen: Brilkas Körper verbindet bruchlos eigene, Familien und Zeitgeschichte. Ob sie das Ballett ihrer Ururgroßmutter Stasia tanzt oder in konvulsivischen Zuckungen an Historie als Krieg und Tod denken lässt: Wo Niza zwischen Erzählerin und Figur gespalten wurde, bleibt Brilka immer Brilka, sodass auch die Tante am Ende des Theaterabends der Nichte befreit zurufen kann: „Tanze, Brilka! Tanze!“

\section{Literatur}

Assmann, AleIDA (2007): Geschichte im Gedächtnis. Von der individuellen Erfahrung zur öffentlichen Inszenierung. München (=Krupp-Vorlesungen zu Politik und Geschichte am Kulturwissenschaftlichen Institut im Wissenschaftszentrum Nordrhein-Westfalen 6).

Costagli, Simone (2010): Family Plots. Literarische Strategien dokumentarischen Erzählens. In: Costagli, Simone / Galdi, Matteo (eds.): Deutsche Familienromane. Literarische Genealogien und internationaler Kontext. München, 157-168.

EIGLER, FRIEDERIKE (2005): Gedächtnis und Geschichte in Generationenromanen seit der Wende. Berlin (=Philologische Studien und Quellen 192).

Freud, Sigmund (1909 / ${ }^{7}$ 1970): Der Familienroman der Neurotiker. In: DERS.: Studienausgabe, Bd. IV: Psychologische Schriften, 221-226.

Galli, Matteo / Costagli, Simone (2010): Chronotopoi. Vom Familienroman zum Generationenroman. In: Costagl, SiMOne / GALli, MATtEo (eds.): Deutsche Familienromane. Literarische Genealogien und internationaler Kontext. München, 7-20.

GEIER, ANDREA (2014): Verstörende Dokumente, irritierende Erzähldynamiken. Potentiale und Probleme des Genres Familienroman (Uwe Timm und Ulla Hahn). In: SÜSELBECK, JAN (ed.): Familiengefühle. Generationengeschichte und NS-Erinnerung in den Medien. Berlin, 129-151.

GiSBERTZ, ANNA-KATHARINA (2018): Die andere Gegenwart. Zeitliche Interventionen in neueren Generationserzählungen, Heidelberg (=Beiträge zur neueren Literaturgeschichte 391).

Halfmann, Roman (2017): Die neue Nostalgie in der Gegenwartskultur. Zur Transformation personaler Authentizität in Werken von Ian McEwan, Karl Ove Knausgård, Nino Haratischwili und Tom McCarthy. In: Weimarer Beiträge 63.1:5-26. 
HARATISCHWILI, NinO (2014): Das achte Leben (Für Brilka). Frankfurt a.M.

JAHN, BERNHARD (2006): Familienkonstruktionen 2005. Zum Problem des Zusammenhangs der Generationen im aktuellen Familienroman. In: Zeitschrift für Germanistik 16.3:581-596.

JELEČ, MARIJANA (2016): Formen der Vergangenheitsbewältigung in ausgewählten zeitgenössischen österreichischen Generationenromanen. In: LOVRIĆ, GORAN / JELEČ, MARIJANA (eds.): Familie und Identität in der Gegenwartsliteratur. Frankfurt a.M. u.a., 147-162.

LÖFFLER, SIGRID (2003): Böse alte Welt. In: Literaturen 07/08:9-16.

KAFKA, Franz (1904 / 1954): Brief an Oskar Pollak vom 27.01.1904. In: Ders.: Gesammelte Werke, [Bd. IX:] Briefe 1902-1924, hg. von Max Brod, Frankfurt a.M. 1958, 27-28. Mannheim, KarL (1928 / 21970): Das Problem der Generationen. In: MANNheIM, KARL: Wissenssoziologie. Auswahl aus dem Werk, eingeleitet und herausgegeben von Kurt H. Wolff. Neuwied a. Rhein / Berlin, 509-565.

ReIDY, Julian (2013): Rekonstruktion und Entheroisierung. Paradigmen des ,Generationenromans ' in der deutschsprachigen Gegenwartsliteratur. Bielefeld (=Figurationen des Anderen. Literatur- und kulturwissenschaftliche Studien 2).

RoHRwASSER, Michael (2003): Das Schwarzbrot der Erinnerung. In: Literaturen 07/08:22-27.

SchÜlKe, ANNE (2016): Früchte des Zorns. Über Gattung und Geschlecht im Kontext des zeitgenössischen Familienromans. In: LOVRIĆ, GORAN / JELEČ, MARIJANA (eds.): Familie und Identität in der Gegenwartsliteratur. Frankfurt a.M. u.a., 119-132.

SILLER, BARBARA (2016): ,Mütterlicherseits ‘ Generationenromane und Geschlechter. In: LOVRIĆ, GORAN / JELEČ, MARIJANA (eds.): Familie und Identität in der Gegenwartsliteratur. Frankfurt a.M. u.a., 41-59.

ŠLIBAR, NEVA (2016): Frauen schreiben Familiengeschichte(n). Von Monika Maron bis Katja Petrowskaja. In: LovRIĆ, Goran/JELEČ, MARIJANA (eds.): Familie und Identität in der Gegenwartsliteratur. Frankfurt a.M. u.a., 15-39.

WeIGel, SigRID (2006): Genea-Logik: Generation, Tradition und Evolution zwischen Kultur- und Naturwissenschaften. München / Paderborn.

WELZER, HARALD (2004): Schön unscharf. Über die Konjunktur der Familien- und Generationenromane. In: Literatur. Beilage zum Mittelweg 36.1:53-64. 